\title{
Surplus Values in Information Ecosystems
}

\author{
Bengt Carlsson and Paul Davidsson \\ Department of Computer Science, Blekinge Institute of Technology \\ Soft Center, 37225 Ronneby, Sweden \\ \{Bengt.Carlsson,Paul.Davidsson\}@bth.se
}

\begin{abstract}
A model of surplus values within information ecosystems is presented. The model is based on the classical definition of surplus value. However, as this definition was developed within a manufacturing industry context, some modifications are necessary to adopt it to the context of information ecosystems, e.g., by taking into account that products are "virtual" rather than physical. Just as in agent-based computational economics, we model economics as evolving systems of autonomous interacting agents in an evolutionary framework. In this way the resulting model is able to capture more dynamic scenarios. The model is formally specified in terms of price, profit, and group gaining functions and is applied to some examples of societies of selfish agents in antagonistic groups to illustrate its dynamic properties. Moreover, we show how the model builds upon labour theory of value and contrast it to consumer value models.
\end{abstract}

Keywords: Multi agent systems, E-commerce, virtual organizations, information ecosystems

\section{Introduction}

What are the similarities and differences between the new digital information society with the old manufacturing society? What underlying conflicts must be dealt with, and how can these conflicts be resolved? The purpose of this article is to investigate the applicability of the surplus value concept within an "information ecosystem" of "infohabitants".

In the remaining part of this chapter, the concept of information ecosystems and the type of antagonistic exploiter-user scenarios that will be studied in this article is introduced. In section 2, a model of surplus values within information ecosystem is presented, which is applied in section 3 to a number of e-commerce application scenarios. A discussion and some conclusions summarize the article.

\subsection{Information ecosystems}

The global information infrastructure is getting more complex each day. In order to cope with this increased complexity, the trends today are to make the infrastructure into a diverse, adaptive, responsive, and open environment. One way of viewing a populated infrastructure is to regard it as an emerging "information ecosystem" of "infohabitants".

The infohabitants are "intelligent" entities, or agents, typically acting on the behalf of humans. Besides being populated by distributed and robust habitants, the ecosystem should be able to adapt to changing conditions, easily scale up or down, and have an openness and universality. This view stems from the similarity between an information ecosystem with infohabitants and a biotic ecosystem [Tansley (1935)] with biological habitants. The structure of biological ecosystems are basically determined by interactions between individuals and the abiotic environment, and by interactions between, different individuals.

The process that shapes the patterns of individuals within an ecosystem is called natural selection. Since natural selection essentially favours the self-interest of indi- 
viduals, group formation within or between species, must hold some advantage for the individual compared to being alone [Wilson (1975), Dawkins (1989), Dennett (1995)]

\subsection{Exploiter-user scenarios}

Within an information ecosystem some activities are performed by infohabitants, or agents, which have conflicting interests. We will here focus on a typical scenario of such an ecosystem where there is one (or more) exploiter agent(s) and one (or more) user agent(s) being exploited. Associated to each of these infohabitants there is a human "owner", who in some way caused the creation of the infohabitant and specified it's goals. Thus, the infohabitant (agent) acts on the behalf of its owner and the owner is responsible for the actions performed by the agent.

The type of situations that will be studied in the article is where a human exploiter wants to make some kind of profit at the expense of the human user by using her exploiter agent against the user agent. The methods for doing this may be, e.g., by selling products or services, or by performing criminal activities.

\subsection{Related work}

We will in what follows extend the traditional surplus value model in order to capture the essential features of information ecosystems. This approach is also in accordance with a biological view of describing ecosystems, where skills and interactions, beside the actual appearance of the habitat, determine the success of the habitants.

Commerce in information goods is offered in widely varying configurations. Kephart et al. present dynamic market interactions focusing on competitive strategies between agents [Kephart et al. (1999)]. Instead of interacting humans, software economic agents will populate the market. Software agents may gain control by incorporating learning behaviour when interacting within the market based agent society [Kephart et al. (2000), Vidal and Durfee (1998)].

In standard labour market models (e.g., [Ehrenberg and Smith (1997)]) a system of demand, supply, and equilibrium equations are used to characterize work suppliers and employers. This approach is compliant with the traditional macro perspective. However, there are also some approaches adopting a micro perspective, such as Agent-based Computational Economics (ACE) [Tesfatsion (1998)], which concerns the computational study of economies modelled as evolving systems of autonomous interacting agents in an evolutionary framework.

A labour market framework [Tesfatsion (1999)] uses a dynamic process model based on individual agents and the interactions between agents instead of a system model like the standard labour market model. In a job-search interaction, worksuppliers and employers engage in a Prisoner's dilemma game where they repeatedly seek worksite partners and evolve worksite behaviours over time.

\section{Surplus values within information ecosystems}

\subsection{The traditional surplus value concept}

Marx introduced the concept of surplus value as a measurement of the profit and a description of the roles of different actors in the production of goods [Marx (1867)]. This is in contrast to the earlier view of increases or decreases in production costs as the only effectors of the rate of profit. Marx was one of the first to describe an economic relation as a formula:

$$
p=c+v+s
$$

where, $\mathrm{p}$ represents the price of the good, c the amount of money spent on investments and material, $\mathrm{v}$ the amount of money spent on labour and $\mathrm{s}$ the surplus value. Here, the unit of money is used as an adequate converter of a time constant to money. 
In a model of work suppliers and employers $\mathrm{c}+\mathrm{v}$ represents the use-value and $\mathrm{c}+\mathrm{v}$ $+\mathrm{s}$ represents the exchange value where the work supplier is supposed to add an extra profit, the surplus value, to the use value.

The surplus value is essential to the dynamics of the system. The manufacturer may invest part of the surplus value in better tools or more effective factories. In an open market where all manufacturers have the same conditions there are typically no other alternatives than making such investments, because otherwise other manufacturers will drive you out of the market. Marx considered the manufacturer and employers as the essential part of a dynamic system, which developed the means of production and increased the amount of produced goods. From a human point of view this is a qualitative increase in the ability to use natural resources.

One important consequence of the surplus value is the biased advantage for the manufacturer against the employer. Marx described a zero-sum game where the concentration of capital for one group was balanced by the loss of resources for the other group. An employee used less and less time for her own needs (because of the more effective production of goods) and more and more time for producing surplus values. The result was highly antagonistic groups (classes).

\subsection{Manufacturing systems versus information ecosystems}

There are different types of resources involved in an information ecosystem depending on whether we are looking at the human or the agent side. The resources on the human side are measured as money and may include transfer of goods or commodities. The resources on the agent side can be described as processor time. An exploiter agent may appropriate some of the user agent's processor capacity for its own purpose. The goal of the interaction between the user agent and the human user is often to prevent privacy abuses.

Within an information system a surplus value appears when some infohabitants profit from other infohabitants. Our approach is to model the resulting group dynamics in an environment of competitive groups of exploiters and users within an information ecosystem. Exploiter agents gain some surplus value benefit at the expense of the user agents. However, there are some differences between a manufacturer system and an information system to take notice of.

- The qualitative use-value factor, expressed as money or time, is hard to give a precise value in an information system because of the lack of physical products. Instead of using a supply-demand description, a recursive iteration of equation (1) will be used when incorporating an information value.

- The accumulation of profit may be different in an information system, it does not have to be increased over time or be connected to the exploiter agents. In both type of system it is only the exploiters that profit from the surplus value. The internal resources typically increase over time, i.e., the systems become more complex, e.g. by the development of more efficient machinery of production and enhanced agent communication.

- A manufacturer produces goods, which have the same use value for all the work suppliers and employers. For infohabitants the exploiters and users can interpret the "produced" information differently. In section 3 we will give an example of mass distribution of e-mail advertisements, interpreted as product information by the exploiters and as "spam-mails" (undesired mass-distributed e-mails) by most of the users.

It can be argued that there are two major strengths and one weakness of the classical theory of surplus values. The first strength is the use of time (normally expressed as money) as the entity for measuring surplus values, because time may be transformed to most other units. For example, processor capacity may be measured by the number of clock cycles during one second.

The second strength is the intrinsic dynamics of the surplus model where individuals within coalitions or between antagonistic groups may try to cooperate or compete. 
One of the main purposes of this paper is to explore this. The weakness is the static concentration of surplus values to one group. Instead of presupposing a dynamical balance between the groups, the distribution of surplus values has to begin all over again, if another group wants to profit by surplus values. Within information ecosystems it seems like the surplus value could be distributed (at least partly) between the different groups. This question will be further analysed in the discussion and conclusion parts.

\subsection{An extended surplus value model for information ecosystems}

We assume an e-commerce system consisting of exploiter and user agents acting on behalf of their human owners. From an e-commerce perspective we may look at an agent as a tool for selling real products or selling/producing virtual products. If we start with the simplification of profit generation in Equation (1), the same formula may be used in a recursive way describing the agent's contribution to the prize of the product. The agents' part, $p_{A}$, of the total prize, $p$, for a product or a duty may be described as:

$$
p_{A}=c_{A}+v_{A}+s_{A}
$$

where $c_{A}$, the constant capital, is investments already made for improving the information ecosystem, $v_{A}$ is a cost arising from "work" done by the agent i.e., facilitating e-commerce trading by interacting with other agents, and $s_{A}$ is the surplus value gained by the exploiter agent. The total prize may now be expressed as:

$$
p=\left(c_{i}+p_{A}\right)+v+s
$$

where $c_{i}+p_{A}$ constitutes the constant capital, and $c_{i}$ represents investments done outside the agents' domain.

An exploiter agent $E$ gets its surplus value $s_{E}=s_{A}$ by doing a work $v_{E}$ plus using the work $v_{U}$ of a user agent $\mathrm{U}$ or $v_{A}=v_{E}+v_{U}$. There are two possible interpretations of this surplus value.

The first interpretation is that the surplus value is part of a selling cost for a "real" product. The work of the exploiter and user agents is included in the total cost of the products just as the surplus value. The role of the exploiter agent is to maximize the success against other exploiters' products and/or increase the total market. If we reduce the function of the agents to handle the distribution of surplus value, instead of creating new values, there will be a zero-sum play describing the total outcome of the e-commerce domain. The purpose for a single exploiter agent is still to maximize the surplus value on behalf of other agents.

The second interpretation is that the surplus value is part of the prize of a virtual product. Some or all surplus value may come from the agent activity (of course basically as a result of a human constructor of the agent). If the function of the agent involves some virtual profit-gaining product, the surplus value increases within a restricted area of e-commerce. There will be an increase in the total surplus value if this new duty does not reduce the value of other duties or products because of cascading activities. The present progress of the Internet should be seen as surplus value extending activities, developing virtual products. This is similar to the development of the manufacturing economy.

The role of the exploiter agent is to facilitate the implementation and increase the surplus value. This latter property may be obtained by more successful acting against user agents and other exploiter agents. In cooperative surroundings this may be done by informing activities, sharing market sites between different agents or whatever move done aiming to extend the market for all agents. Another, more important, diverging role is to compete against other agents. This competition may result in both 
gains and losses of surplus values for an exploiter agent both against other exploiter agents and user agents.

The role of the surplus value is to be a catalyst for maximizing profit against other antagonistic participants. This is partly in accordance with an evolutionary model based on natural selection. Exploiter and user agents constitute different antagonistic groups within information ecosystems i.e., they have contrary to biotic systems biased the utility function toward the exploiter group.

A supply-demand description of profit generation does explain a regulator of different interests. As long as undesired varieties, like monopolization, are taken care of the origin of common good will still be present. The simplified surplus value formulas may be used to focus on the catalyst quality, but the interpretation of an information ecosystem case has to be done with respect to a specific domain. Most details of such an interpretation is outside the scope of this work. In another work [Carlsson and Gustavsson (2001)], a protection mechanism for a computer security domain, based on exposure time, against intruders, further analysing the concept of surplus values has been proposed. The intruders try to maximize their surplus values against other intruders or regular users. In the next section we examine a case of mass distribution of e-mail advertisements.

\section{Application of the extended surplus value model}

Whenever a user uses the Internet she exposes herself to a potential risk of privacy abuse. For instance, by downloading files containing music, the titles may secretly be recorded to create a profile of the musical taste. There are companies selling personal information, so-called investigative services and "spywares". Mail-order firms are tracking the products you buy, consumer profiling, and sell lists to third-party marketers. It is fairly simple to keep a record of every site a user agent visit and every transaction made. For example, when visiting a Web page with a certain banner a cookie can be deposited on the user's hard drive. Then any time a page containing this banner is visited, the cookie on the hard drive is used to send information about what sites on the Internet (some or all) that the user has visited. PC World concluded in a recent cover story about privacy:

"In fact, the biggest threat to your privacy today isn't crackers, stalkers, or data brokers. It's the legitimate online business - such as advertising networks, retailers, and others - that are creating detailed profiles of who you are and what you do when you are on the Web." [Tynan 2000]

In addition, there are a variety of more or less criminal methods involving, e.g., credit card deception, usage of other users' processor capacity, or illegal use of copyrighted material. Whether or not a certain activity should be regarded as exploitative or not has to be decided from case to case. In the following examples our intention is to show how a surplus value model can be used to explain the underlying conflict between the actors within e-commerce.

\subsection{Popularising trademarks on the net}

Today there are millions of web sites reachable by any Internet user. To be visible, ebusiness and other companies have to spend a lot of money on creating brand recognition on the net. This money must sooner or later generate profit, or surplus values, to the companies. The companies use an exploiter agent, probably built into a web site. The user agent spends time visiting this site with direct or indirect instructions from the user. When buying something, the user will pay the price of the goods, which also includes a surplus value for the company directing the buyer to the desired product. 
A successful seller has a web site visited by many potential buyers. Unlike the traditional surplus value model within manufacturing business, a lot of capital for building a web site is not needed but maybe for keeping it as a successful site. This means that the potential number of e-business companies hardly will be reduced; the risk of getting a monopoly situation in a free market is negligible. Companies advertising too much or having too high prizes will not survive.

The buyers have the opportunity to compare lots of different sites visited. When buying a product the user agent, a "shop-bot", will probably visit a number of sites and compare prices, whereas the (human) user may turn about the quality of the company. It is in the interest of the company to offer the users their "user agent". Such agents may put the company in a top position when doing a search or offer spin-off products not yet requested. The users, because of lack of reliability, will avoid a too "efficient" agent, i.e. an agent maximizing a certain company's interest. The difference between a user and an exploiter "user agent" will be hard to recognize for the human buyers.

\subsection{Mass distribution of e-mail advertisements}

In this scenario there are two types of infohabitants: "spam agents" on the exploiter side and filtering agents on the user side. The task of a spam agent is to send e-mail advertisements for products that its owner sells to as many persons as possible. The human owners spend time mainly on reading/writing and addressing e-mails, but some time may also be spent interacting with their agents.

The goal of the exploiter is to save money (e.g., lowering advertising costs) by sending spam mails. It is assumed that a few users react positively to the exploiter's messages (and consequently do not consider the message as a spam mail). However, for the users as a whole, the messages from the exploiters should still be considered as spam mails. The messages are not directed to a specific group of interested users and on average the disadvantage of receiving the messages is larger than the advantage of getting the product information that may be included in the message.

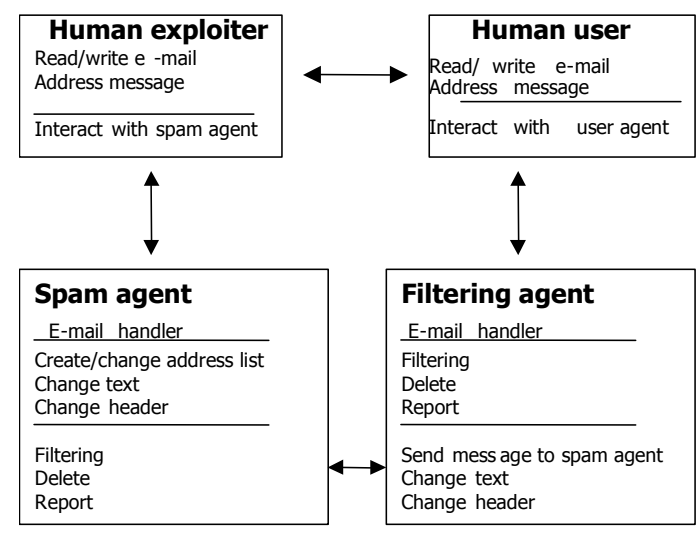

Figure 1. An e-mail information ecosystem consisting of humans, spam agents, and filtering agents.

The conflict between the exploiter and user groups is antagonistic; what one part gains the other will lose. The main goal for the spam agents is to increase the number of (spam) messages read by the users, e.g., by sending as many e-mails as possible that are not filtered out by the filtering agents. In order to achieve its goal a successful spam agent may have to invest in better address lists, better editing facilities, and even to defend from filtering agents' counterattacks. The main goal for the filtering agent is to prevent the spam messages from reaching the user. It may also inform the 
user about incoming spam messages and, together with the user, decide upon actions against future spam agent attacks.

Using the Equation (2) the $v_{A}$ part consists of cost for work done by the exploiter agent $v_{E}$ and the work done by the user agent $v_{U}$. The surplus value $s_{E}$ wholly belongs to the exploiter agent resulting in

$$
p_{A}=c_{A}+v_{E}+v_{U}+s_{E}
$$

The $v_{E}$ cost include all handling costs for sending the spam mail like create/change address lists, change text and change header (see Figure 1). The cost of $v_{U}$ includes all the activities of the filtering agent like doing the detection of spam-mails, deleting spam mails and reporting to the user. The $v_{U}$ cost also includes the handling cost for those mails passing the filtering function.

Suppose that on average a filtering agent has a success-rate $\alpha$ detecting and removing the spam-mails. Let us call the exploiter agents handling cost $A$, the filtering agents handling cost $B$, and the handling cost of successful spam-mails $C$. A benefit $\mathrm{D}_{n}$, for the exploiter $n$, is supposed for delivering a spam mail. We also suppose a proportion $\beta$ of the spam-mails reaching the user corresponding to how many of the successful messages sent by the exploiter agents that are of actual interest for the user. We are supposing a variable success rate from exploiter to user agents and a fixed rate of interest among the users (at a rough estimate around $1 \%$ for spam mails and $0 \%$ for viruses). The average cost for a group of $N$ exploiter agents each sending $x_{n},(n=1, \ldots, N)$ spam-mails and the corresponding user agents is:

$$
v_{A}=\frac{\sum_{n=1}^{N}\left(N A+x_{n} \alpha B+x_{n}(1-\alpha) C\right)}{N}
$$

The average surplus value for the exploiter agents is:

$$
S_{E}=\frac{\sum_{n=1}^{N}(1-\alpha) \beta_{n} x_{n} D_{n}}{N}
$$

If the number of spam mails per user increases, this is a drawback for the filtering agents and the users. The filtering agents have to work harder filtering out spam messages and the likelihood for a spam mail reaching the users increases. An increased number of spam agents does not affect the surplus value for a single spam agent, but increases the cost for every filtering agent.

In our example, the dynamics of the groups will consist of interaction between the groups followed by interaction within each group. A spam agent should not rely too heavily on other members of the group. Despite a few advantages of belonging to a group of spam agents, the disadvantages dominate. Why improve the tools for sending spam messages together with other agents if the risk of being outdone increases heavily?

Cooperation within the filtering agent group will be favoured because everyone has something to gain from getting together. Better tools against spam agents can be developed which identify spam mails easier (since the task is distributed). Possibly all spam agents will be wiped out, but the system is not robust. Nothing prevents a new agent from becoming a spam agent in the future or an agent from leaving the filtering agent group.

The surplus value means that the user agent has to deliver extra messages to the user i.e. add some extra cost for using ordinary information. The exploiter agent only has to receive one package of information from the agent owner, but is able to send the information to several user agents, i.e. by increasing the internal resources the surplus value multiplies. 
If the user agents increase their internal resources by acting together against the exploiters, the surplus value will decrease, i.e. the success rate $\alpha$ decreases. The motive power for making $\alpha$ as small as possible is an underlying creation of surplus values for some of the users, i.e. becoming exploiters and leaving the group of users. A more effective filtering tool is a surplus value generating virtual product, which some former users may introduce. This will be further analysed below.

\section{Discussion}

In Section 2.2, we argued that the generation of surplus values might be distributed between different groups. However, looking back at the spam agent example, this was not the case because a user getting a surplus value will automatically become an exploiter. The main difference between information ecosystems and manufacturing factory systems is rather in the distribution of user and exploiter groups than in the definition of the groups.

The most important difference between the new information economy and the traditional economy is probably that the production tools are already in the hands of the users. There is no need for owning a factory; a standard personal computer is often sufficient for developing the necessary software or web pages. We are not denying the benefit of a strong infrastructure, but it is the intellectual capital that is the most important. Another difference is that in the information economy, the surplus value concept typically goes beyond making direct profit. For example, there may be a surplus value involved in being "famous", that can be achieved by creating a muchused freeware program or a well-attended home page. This fame may be transformed into profit later on.

In the example of sending spam mails a surplus value consisting of a success rate and a rate of interest was introduced. The success rate may decrease for the exploiter agent if a group-gaining factor like a tool for collaborative filtering increases the success against the group of exploiter agents.

Instead of having a decreasing group of exploiters maximizing increasing surplus values, we may end up with an increasing group of former users making their own surplus values (on the behalf of the other users and/or exploiters). These new tools or services broaden the domain considered and explain the origin of new surplus values.

The improvements of the exploiter and user groups may result in an arms race. A virus collecting login names may be spread as a program among a group of hacker agents. This will cause the users to install protection software recognizing this type of virus. One improvement by the hacker agent is retorted by a counter-improvement from the anti-virus agent, so in the long run the hacker will meet a better-protected user.

There are three cases concerning the group dynamics of the exploiter agents. The average surplus value may increase, remain the same, or decrease.

1. If the surplus value increases, the group of exploiter agents becomes stronger. This in turn makes the internal resources increase because of the accumulation of surplus values. However, the opposite is not true. The amount of internal resources for an exploiter will not automatically increase the surplus value because the users may also increase its internal resources. An accumulation of internal resources says nothing about the success for a single exploiter agent. Due to increased competition between exploiters there is a possibility for a few agents to dominate the other exploiters. For this example an increased competition within the user agent group may occur or cause non-cooperating user agents to disappear because of increased pressure from the successful exploiter agents. There will be an arms race within the users where less successful behaviours will go extinct. 
2. If the surplus value is not changing there will be a balance between exploiter and user agents. Actions from one group are balanced by counter-actions from the other group.

3. If the surplus value decreases, the group of exploiters becomes weaker. With increased competition the number of exploiters decreases, i.e., the successful exploiters improve their methods, and the less successful become user agents.

Generally speaking an exploiter $E_{x}$, with $s_{E_{x}}>\overline{s_{E}}$, where $\bar{S}_{E}=\frac{1}{N} \sum_{i=1}^{N} s_{E_{i}}$, may use these extra resources to gain control of the information passing while an exploiter $E_{y}$, with $s_{E_{y}}<\overline{s_{E}}$ will lose some or all of its exploitation capabilities. The sum of all actions taken by the different exploiter agents is a zero sum game. The surplus value will enhance the competition within the group of exploiters, making some of them stronger while other will vanish. Unlike a manufacturer system of accumulated constant capital there does not have to be a concentration of internal resources in the information ecosystem.

In the popularisation on trademarks, there is a possibility of having successful companies getting out of the business instead of having e-trading companies getting stronger. To be successful for an exploiter agent means having "control" of user agents. This control function may be regulated by laws, proprietary formats or, as in the trademark example, by developing manipulating search engines.

\section{Conclusions}

The dynamics of the group of exploiter agents may be characterised by at least three different aspects:

- An ambition to increase the surplus value - this will make the $s_{\text {avg }}^{E}$ increase and/or the number of exploiter agents decrease.

- Forming new exploiters - the exploiters' new agents will prevent previous exploiters from monopolizing the net and possibly decrease the surplus value of the previous exploiter agents.

- Synergetic effects - because of the similarity in means of production for the exploiter agents it is easy to enlarge the domain of the ecosystem resulting in a total increased surplus value.

The dynamics of the human exploiters include traditional manufacturing business, monopolizing resources when taking over competitors, making laws and security instructions. These moves may have a tremendous influence on the exploiter agents, but it is outside the scope of this work to explicitly point out all the details involved.

The e-commerce examples show that two antagonistic groups of selfish agents may rely on each other even if one of them is a destructive group. This has to do with the benefit for a single agent against another group or in conjunction with its own group. Only robust groups of agents are able to handle an attack from a malicious agent well, by evolving countermeasures against the intruder. This evolving process is facilitated by the possibility for a user to receive a surplus value without its agent becoming an exploiting agent in the current domain. As an example a filtering program may make money for its owner without being an exploiting spam agent. On the other hand, a user's search agent may act in the interest of an exploiter, making it an exploiter agent as the trademark example points out.

The reported work of surplus values introduced here, is part of a series of studies of different aspects of surplus values and antagonistic behaviours within information ecosystems. Current examples which may be further used and developed are:

- A tool for analysing very large groups like the actors on Internet. It may be possible to find patterns for the behaviours of agents in different antagonistic groups. 
In particular we may find robust solutions when information ecosystems are modelled as biological ecosystems [Carlsson and Davidsson (2001)],

- A tool for implementing a society of selfish agents in antagonistic groups. A well defined domain of the agents is presupposed. From the initial assumption of a homogenous group of agents, it is possible to model how antagonistic groups try to reach a predefined goal. This could be maximal surplus value for the manufactures or maximal robustness for the users. An arms race between antagonistic participants using more and more refined agents is a plausible outcome [Carlsson (2001)].

- An example of interactions based on the dynamics of security and integrity systems. In Carlsson and Gustavsson [2001] a model based on time based security [Schwartau (1999)] is further analysed from a surplus value perspective.

\section{Acknowledgements}

We would like to thank Magnus Boman, Ingemar Jönsson and the anonymous reviewers for their comments on various drafts of this work and Martin Hylerstedt for proof reading.

\section{References}

Carlsson, B. (2001) The Tragedy of the Commons - Arms Race within Peer-to-Peer Tools. To appear in the proceedings of the 2nd International Workshop Engineering Societies in the Agents' World. ed. Omicini, A., Petta, P., and Tolksdorf, R. Lecture Notes in Artificial Intelligence 2203, Springer-Verlag.

Carlsson, B. and Davidsson, P. (2001) A Biological View on Information Ecosystems. To appear in the proceedings of the Second Conference on Intelligent Agent Technology, World Scientific.

Carlsson, B. and Gustavsson, R. (2001) Arms Race Within Information Ecosystems. To appear in the proceedings of the Fifth International Workshop Cooperative Information Agents, Springer-Verlag.

Dawkins, R.(1989) The Selfish Gene. 2nd edn. Oxford University Press, Oxford. Dennett, D.C. (1995) Darwin's Dangerous Idea Allen Lane Penguin Press, London. Ehrenberg, R.G., Smith. R.S. (1997) Modern Labor Economics: Theory and Public Policy. 6th edn. Addison-Wesley, Reading, MA.

Kephart, J.O., Das, R. and MacKie-Mason, J.K. (2000) Two-sided Learning in an Agent Economy for Information Bundles. In Agent-mediated Electronic Commerce, Lecture Notes in Artificial Intelligence, Berlin, Springer-Verlag.

Kephart, J.O., Hanson, J.E. and Greenwald, A.R. (1999) Dynamic Pricing by Software Agents Computer Networks vol 32-6 731-752.

Marx, K. (1867) Capital volume 1 Penguin books, London 1976.

Schwartau W. (1999) Time Based Security. Practical and Provable Methods to Protect Enterprise and Infrastructure, Networks and Nation Interpact Press.

Tansley, A.G. (1935) The Use and Abuse of Vegetational Concepts and Terms, Ecology 16 284-307.

Tesfatsion, L. (1999) Structure, Behavior, and Market Power in an Evolutionary Labor Market with Adaptive Search. ISU Economic Report No. 51.

Tesfatsion, L. (1998) Teaching Agent-Based Computational Economics to Graduate Students. ISU Economic Report No. 45.

Tynan D. (2000) Privacy 2000 - In Web We Trust? PC World June 103-116.

Vidal, J.M. and Durfee, E.H. (1998) The Moving Target Problem in Multi-agent Learning. In Proceedings of the Third International Conference on Multi-Agent Systems (ICMAS98).

Wilson, E.O. (1980) Sociobiology - The abridged edition. Belknap Press, Cambridge. 